\title{
Functional diversity of miR-146a-5p and TRAF6 in normal and oral cancer cells
}

\author{
SEUNG-KI MIN ${ }^{1,2}$, SUNG YOUN JUNG ${ }^{3}$, HYUN KI KANG $^{3}$, SIN-A PARK $^{3}$, \\ JONG HO LEE ${ }^{2}$, MYUNG-JIN KIM ${ }^{2}$ and BYUNG-MOO MIN ${ }^{3}$ \\ ${ }^{1}$ Oral Oncology Clinic, Research Institute and Hospital, National Cancer Center, \\ Goyang-si, Gyeonggi-Do 10408; ${ }^{2}$ Department of Oral and Maxillofacial Surgery and \\ ${ }^{3}$ Department of Oral Biochemistry and Program in Cancer and Developmental Biology, Dental Research Institute, \\ Seoul National University School of Dentistry, Seoul 03080, Republic of Korea
}

Received May 24, 2017; Accepted September 6, 2017

DOI: 10.3892/ijo.2017.4124

\begin{abstract}
Numerous studies implicate miR-146a as pleiotropic regulator of carcinogenesis; however, its roles in carcinogenesis are not fully understood. A clue from expression analyses of miR-146a-5p in all 13 oral squamous cell carcinoma (OSCC) cell lines examined and in OSCC tissues, whole blood and whole saliva of OSCC patients in vivo revealed that miR-146a-5p expression was highly upregulated. Particularly, we widened the view of its upregulation in saliva, implicating that high miR-146a-5p expression is not only correlated closely to the development of human oral cancer, but also to a possible candidate as a diagnostic marker of OSCC. Indeed, further examination showed that exogenous miR-146a-5p expression showed pleiotropic effects on cell proliferation and apoptosis which were partially based on the contextual responses of activation of JNK, downstream of TRAF6 that was targeted by miR-146a-5p in normal human keratinocytes and OSCC cell lines. TRAF6 suppression by a TRAF6-specific siRNA resulted in contradictory consequences on cellular processes in normal and OSCC cells. Notably, TRAF6 downregulation by both miR-146a-5p and TRAF6-specific siRNA deactivated JNK in SCC-9, but not in normal human keratinocytes. In support of the proliferation-promoting effect of miR-146a-5p, silencing of endogenous miR-146a-5p significantly reduced proliferation of SCC-9. Together, these results suggest that
\end{abstract}

Correspondence to: Professor Byung-Moo Min, Department of Oral Biochemistry and Program in Cancer and Developmental Biology, Seoul National University School of Dentistry, 101 Daehak-Ro, Jongno-Gu, Seoul 03080, Republic of Korea

E-mail: bmmin@snu.ac.kr

Professor Myung-Jin Kim, Department of Oral and Maxillofacial Surgery, Seoul National University School of Dentistry, 101 Daehak-Ro, Jongno-Gu, Seoul 03080, Republic of Korea

E-mail: myungkim@snu.ac.kr

Key words: oral squamous cell carcinoma, miR-146a-5p, cellular process, TRAF6, JNK
miR-146a-5p affects proliferation and apoptosis in a cellular context-dependent manner and selectively disarms the TRAF6-mediated branch of the TGF- $\beta$ signaling in OSCC cell lines by sparing Smad4 involvement.

\section{Introduction}

MicroRNAs (miRNAs) are small non-coding RNAs implicated in post-transcriptional suppression of target genes in a sequence-specific manner by suppressing mRNA translation and, less frequently, causing degradation of the target mRNA (1). They have been implicated in the regulation of as many as $30 \%$ of the human cell mRNAs, with fundamental roles in cell proliferation, differentiation and apoptosis. A single miRNA molecule has the capacity to target a wide range of different mRNAs, some of which may have opposing oncogenic or tumor-suppressive functions, depending on the context (2). Furthermore, the growth and spread of a cancer is both the combinations of a function of the cancer cells themselves and various extrinsic factors, which interact with the cancer cells, affecting their behavior. miRNAs influence largely on each of the extrinsic factors such as the immune system, tumor stromal cells, therapy and oncoviruses (2).

Several studies implicate miR-146a as pleiotropic regulator of carcinogenesis, as polymorphism and altered expression have been linked with cancer risk and invasive and metastatic capacity in diverse cancers (3); however, its roles in carcinogenesis are not fully understood. miR-146a-5p has been reported to be overexpressed in squamous cell carcinoma (SCC) of the head and neck, cervix and lung (4-6), and is implicated in oncogenicity of oral squamous cell carcinoma (OSCC) (7). In vitro studies indicate that transfection of miR-146a precursor results in cell proliferation (5); additionally, transient transfection of a miRNA-146a mimic into human bronchial epithelial cells can protect the cells from apoptosis in response to the combination of inflammatory cytokines and transforming growth factor- $\beta 1$ (TGF- $\beta 1$ ). However, it is suppressive in many other malignancies, including breast, gastric, prostatic and pancreatic carcinomas (8-11). In fact, miR-146a seems to play multiple roles in the regulation of different phenotypes by targeting a wide range of different genes in various 
cellular contexts, leading to the controversy to its contribution in cancer. Emerging evidence suggests that there is a direct link between miRNAs and cancer (12), as well as, inflammation $(13,14)$. Chronic inflammation and the activation of nuclear factor kappa $\mathrm{B}(\mathrm{NF}-\kappa \mathrm{B})$ are known to be associated with development of multiple cancers (15), while inflammatory cytokines are potent stimulants of miR-146a through $\mathrm{NF}-\kappa \mathrm{B}$ pathway $(16,17)$. Specifically, several studies report that various miRNAs, including hsa-miR-20a, hsa-miR-142-3p, hsa-miR-19a, hsa-let-7f, hsa-miR-203, hsa-miR-17, hsa-miR223, hsa-miR-146b and hsa-miR-146a, are highly expressed in patients with periodontitis (18-20). Furthermore, through regulation of interleukin-6 (IL-6)/signal transducer and activator of transcription 3 (STAT3) signaling, miR-146a may link the chronic inflammation and cancer (21).

Notably, miR-146a-5p has been shown to target Smad4 (22) and tumor necrosis factor receptor-associated factor 6 (TRAF6) (23), which both serve as significant mediators in the TGF- $\beta$ pathway. TGF- $\beta$ regulates a wide array of cellular processes such as cell growth, differentiation, apoptosis, migration and extracellular matrix production (24). It is a potent antitumor cytokine because of its property as a strong inhibitor for the growth of epithelial cells. TGF- $\beta$ utilizes multiple different signaling pathways in addition to the canonical Smad pathway (25). These non-canonical pathways include mitogenactivated protein kinase (MAPK) pathways, downstream of TRAF6. TGF- $\beta$ receptors interact with TRAF6 and induce the formation of K63-linked poly-ubiquitin chains on TRAF6, recruiting TGF- $\beta$-activated kinase 1 (TAK1) to activate Jun N-terminal kinase (JNK) and p38 MAPK (p38) (26,27). Signal integration between JNK and p38 determines cell type-specific and context-dependent proliferation, survival and differentiation of human tumors and cancer cell lines (28).

TRAF6 plays important roles in the transcription and expression of numerous inflammation- and apoptosis-related genes (29). TRAF6 is known to promote apoptosis by activation of caspase-8 (30), similarly to the action of Fas associated death domain (FADD) on stimulation by Fas (31). Compellingly, FADD is also a target gene suppressed by miR-146a-5p and the suppression is related to resistance against activation-induced cell death (32). The third domain of FADD contains the phosphorylation site at Ser194 residue partly contributing to the activities of FADD in humans (33), and phosphorylated FADD is translocated into the nucleus to perform apoptosis unrelated functions (34).

In the present study, we demonstrated that miR-146a-5p, which is frequently upregulated in OSCC cell lines and in blood and saliva of OSCC patients compared with normal counterparts, showed pleiotropic effects on proliferation and apoptosis of OSCC cell lines. This was partially based on the contextual responses of JNK, downstream of TRAF6 that was suppressed by miR-146a-5p in SCC cell lines. Our data suggest that miR-146a-5p affects cellular processes in a cellular context-dependent manner, and potentiates proliferation of OSCC cell lines.

\section{Materials and methods}

Cultures of primary human cells and OSCC cell lines. Primary normal human oral keratinocytes (NHOKs) were prepared and maintained as previously reported (35). Briefly, NHOKs were isolated from human gingival tissue specimens obtained from healthy volunteers (age range, 20-30 years) who were undergoing oral surgery. NHOKs were cultured in keratinocyte growth medium containing $0.15 \mathrm{mM}$ calcium and a supplementary growth factor bullet kit (KGM; Clonetics, San Diego, CA, USA). Keratinocytes in their second passage were used in the described experiments. Primary normal human dermal fibroblasts (NHDFs) were prepared and maintained as previously described (36). The HOK-16B line, human oral keratinocytes immortalized by transfecting cells with the cloned HPV-16 genome (37), was also cultured in KGM. The cancer cell lines SCC-4, SCC-9, FaDu and SiHa were purchased from the American Type Culture Collection (ATCC; Rockville, MD, USA). SCC-4 and SCC-9 cell lines were cultured in Dulbecco's modified minimum essential medium (DMEM)/Ham's F12 (Gibco-BRL, Bethesda, MD, USA) supplemented with $10 \%$ fetal bovine serum (FBS) and $0.4 \mu \mathrm{g} / \mathrm{ml}$ hydrocortisone. Among three oral cancer cell lines, CTHOK-16B-BaP and CTHOK-16B-DMBA, which were tumorigenically transformed by chronic exposure of the HOK-16B cells to benzo(a)pyrene and 7,12-dimethylbenz $(a)$ anthracene, respectively (38), and Spt-HOK80, which was spontaneously transformed from primary human oral keratinocytes (39) were cultured in DMEM supplemented with $10 \%$ FBS and $0.4 \mu \mathrm{g} / \mathrm{ml}$ hydrocortisone. Cell lines such as CTHOK-16B-DMBA and Spt-HOK80 were established by our group in 1995 and 2010, respectively $(38,39)$. HOK-16B-BaP cell line, the original name of which is CTHOK-16B-BaP, was first reported by our group in 1995 (38). Other oral cancer cell lines KOSCC-11, KOSCC-25A, KOSCC-25B, KOSCC-25C, KOSCC-25D, KOSCC-25E, KOSCC-33A and KOSCC-33B (40) were also cultured in DMEM supplemented with $10 \%$ FBS and $0.4 \mu \mathrm{g} / \mathrm{ml}$ hydrocortisone. The SiHa line was grown in DMEM supplemented with $10 \%$ FBS, while the FaDu line was grown in DMEM supplemented with $10 \%$ FBS and $0.4 \mu \mathrm{g} / \mathrm{ml}$ hydrocortisone.

Blood, saliva and OSCC tissue samples. The whole blood, whole saliva and OSCC tissue samples used in this study were obtained from OSCC patients who underwent surgical resection at the Department of Oral and Maxillofacial Surgery, Seoul National University Dental Hospital, and healthy controls who did not have signs or symptoms of inflammation of the gingival tissues and systemic diseases. In the present study, the oral hygiene of oral cancer patients was not taken into consideration. This was because OSCC patients with poor oral hygiene followed by chronic inflammation, including periodontitis, have high incidence of oral cancer clinically, making it difficult to exclude patients with inflammation. All procedures for obtaining human gingival tissue specimens, whole blood, whole saliva and cancer specimens were reviewed and approved by the Institutional Review Board on Human Subjects Research and the Ethics Committee at Seoul National University Dental Hospital.

RNA isolation in the whole blood, saliva and cells. Four hundred microliters of the whole saliva were used for RNA isolation. Saliva samples were extracted using the mirVana ${ }^{\mathrm{TM}}$ miRNA Isolation kit (Ambion, Austin, TX, USA) according 


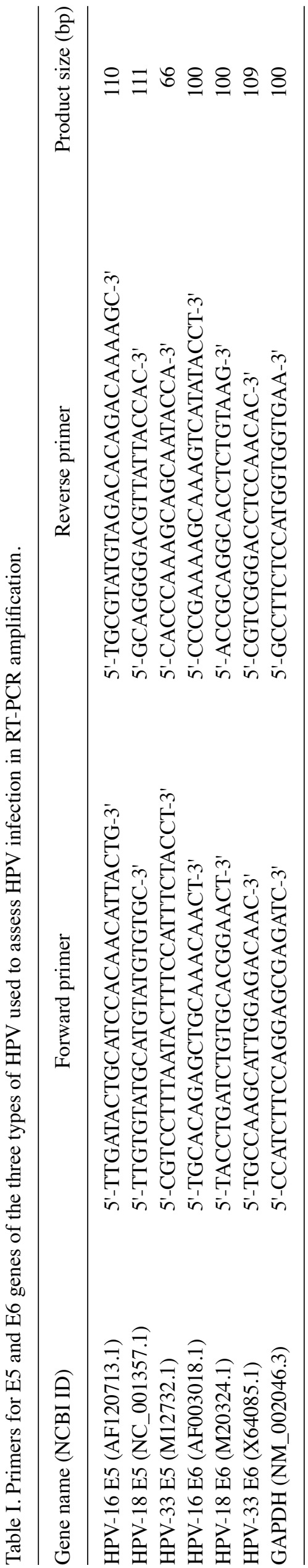

to the manufacturer's instructions. For the initial lysis step, we used $1 \mathrm{ml}$ of lysis/binding solution per $400 \mu \mathrm{l}$ saliva sample. Total RNAs including small RNAs were isolated in whole blood and cells by miRNeasy Mini kit (Qiagen, Valencia, CA, USA) according to the manufacturer's instructions.

miRNA microarray. Cyanine 3-labeled complementary RNA was prepared from total RNA including miRNA (500 ng) using Agilent's Low RNA Input Linear Amplification kit (Agilent Technologies, Santa Clara, CA, USA). Labeled cRNA was applied to microarray (Human miRNA Microarray Release 19.0, 8x60K; Agilent Technologies) using Agilent's Gene Expression Hybridization kit. Hybridized microarray was washed using Agilent's Gene Expression Wash Buffer kit. The microarray chip was scanned using Agilent's DNA microarray scanner, and the raw signal density was acquired from Feature Extraction software. The differential gene expression was classified by Benjamini-Hochberg's false discovery ratio method using GeneSpring GX12 software.

TaqMan miRNA assay. Complementary DNAs (cDNAs) were synthesized from total RNA including miRNA using TaqMan ${ }^{\circledR}$ MicroRNA reverse transcription kit with genespecific primers (Applied Biosystems, Foster City, CA, USA). Reverse transcription reactions (for final quantity or concentrations) contained $10 \mathrm{ng}$ RNA samples, $1 \mathrm{mM}$ of dNTPs, 1X RT primer, 1X RT buffer, $3.8 \mathrm{U}$ of RNase inhibitor and $50 \mathrm{U}$ of reverse transcriptase. The $15 \mu \mathrm{l}$ reactions were incubated for $30 \mathrm{~min}$ at $16^{\circ} \mathrm{C}, 30 \mathrm{~min}$ at $42^{\circ} \mathrm{C}, 5 \mathrm{~min}$ at $85^{\circ} \mathrm{C}$ and then held at $4^{\circ} \mathrm{C}$. Quantitative real-time PCR (RT-qPCR) quantification of miRNA expression was carried out using TaqMan ${ }^{\circledR}$ MicroRNA assays kit (Applied Biosystems) according to the manufacturer's instructions. The primers used were: miR-146a-5p (ID, 000468) and RNU44 (ID, 001094) (Applied Biosystems). The $20 \mu \mathrm{l} \mathrm{PCR}$ included $1.33 \mu \mathrm{l}$ RT product, 1X TaqMan ${ }^{\circledR}$ Universal Master Mix II without UNG, and 1X TaqMan-primers mix (Applied Biosystems). Reactions were incubated in a 96 -well plate at $95^{\circ} \mathrm{C}$ for $10 \mathrm{~min}$, followed by 40 cycles of $95^{\circ} \mathrm{C}$ for $15 \mathrm{sec}$ and $60^{\circ} \mathrm{C}$ for $1 \mathrm{~min}$. The threshold cycle $(\mathrm{Ct})$ was determined using default threshold settings. All experiments were done in quadruples. The RNU44 was used as a control to normalize miRNA input in the RT-qPCR assay.

Determination of HPV infection. The presence of 'high-risk' HPV (type 16, 18 and 33) DNA was determined by reverse transcription polymerase chain reaction (RT-PCR). Total RNA was reverse transcribed to cDNA, which was used for PCR amplification of HPV E5 and E6 (38 cycles) and GAPDH (28 cycles; denaturation at $95^{\circ} \mathrm{C}$ for $20 \mathrm{sec}$, annealing at $60^{\circ} \mathrm{C}$ for $10 \mathrm{sec}$ and extension at $70^{\circ} \mathrm{C}$ for $10 \mathrm{sec}$ ). The primers (Table I) amplified the E5 and E6 genes of the three types of HPV. The PCR products were resolved on a $1.5 \%$ agarose gel, stained with ethidium bromide and visualized using a UV illuminator.

Transfection. A non-specific control siRNA (Invitrogen, Carlsbad, CA, USA) and siRNAs against human TRAF6 (40 nM; Sigma-Aldrich, St. Louis, MO, USA) and FADD (40 nM; Santa Cruz Biotechnology, Santa Cruz, CA, USA) were used for gene silencing. To regulate miR-146a-5p expression in cells, specific miRVana mimic, inhibitor (Ambion) and 
Table II. miRNA expression profile in HPV-16-immortalized human oral keratinocytes relative to the NHOKs by miRNA microarray.

\begin{tabular}{|c|c|c|c|c|c|}
\hline \multicolumn{2}{|c|}{ Upregulated miRNAs } & \multicolumn{4}{|c|}{ Downregulated miRNAs } \\
\hline Systematic name & Fold change & Systematic name & Fold change & Systematic name & Fold change \\
\hline hsa-miR-3934-5p & 15214.5 & hsa-let-7d-5p & 0.50 & hsa-let-7e-5p & 0.35 \\
\hline hsa-miR-365b-5p & 415.0 & hsa-miR-365a-3p & 0.49 & hsa-let-7g-5p & 0.34 \\
\hline hsa-miR-146a-5p & 391.2 & hsa-miR-130a-3p & 0.49 & hsa-miR-5100 & 0.33 \\
\hline hsa-miR-3138 & 282.7 & hsa-miR-4286 & 0.48 & hsa-let-7a-5p & 0.31 \\
\hline hsa-miR-4430 & 6.4 & hsa-miR-18a-5p & 0.48 & hsa-let-7i-5p & 0.31 \\
\hline hsa-miR-150-3p & 5.8 & hsa-miR-4306 & 0.48 & hsa-miR-1260b & 0.31 \\
\hline hsa-miR-378d & 4.1 & hsa-miR-20a-5p & 0.46 & hsa-let-7f-5p & 0.29 \\
\hline hsa-miR-30b-3p & 3.7 & hsa-miR-99a-5p & 0.45 & hsa-miR-4284 & 0.28 \\
\hline hsa-miR-6068 & 3.2 & hsa-miR-30c-5p & 0.45 & hsa-let-7c & 0.28 \\
\hline hsa-miR-1275 & 3.0 & hsa-miR-455-3p & 0.44 & hsa-miR-21-5p & 0.28 \\
\hline hsa-miR-378i & 3.0 & hsa-miR-374b-5p & 0.43 & hsa-miR-455-5p & 0.27 \\
\hline hsa-miR-378a-3p & 2.9 & hsa-miR-31-3p & 0.42 & hsa-miR-29b-3p & 0.26 \\
\hline hsa-miR-30d-5p & 2.8 & hsa-miR-185-5p & 0.41 & hsa-miR-1260a & 0.25 \\
\hline hsa-miR-1229-5p & 2.7 & hsa-miR-28-5p & 0.41 & hsa-miR-26b-5p & 0.25 \\
\hline hsa-miR-5787 & 2.7 & hsa-miR-222-3p & 0.40 & hsa-miR-125b-5p & 0.24 \\
\hline hsa-miR-3679-5p & 2.7 & hsa-miR-4465 & 0.39 & hsa-miR-96-5p & 0.24 \\
\hline hsa-miR-4787-5p & 2.6 & hsa-miR-210 & 0.37 & hsa-miR-374a-5p & 0.23 \\
\hline hsa-miR-205-3p & 2.5 & hsa-miR-29a-3p & 0.37 & hsa-miR-98-5p & 0.21 \\
\hline hsa-miR-6724-5p & 2.4 & hsa-miR-221-3p & 0.37 & hsa-miR-30a-5p & 0.20 \\
\hline hsa-miR-181a-5p & 2.2 & hsa-miR-3659 & 0.37 & hsa-miR-1273g-3p & 0.16 \\
\hline hsa-miR-638 & 2.2 & hsa-miR-203a & 0.37 & hsa-miR-100-5p & 0.10 \\
\hline hsa-miR-4739 & 2.1 & hsa-miR-424-5p & 0.37 & hsa-miR-135b-5p & 0.08 \\
\hline hsa-miR-2861 & 2.0 & hsa-let-7b-5p & 0.36 & hsa-miR-30a-3p & 0.00 \\
\hline
\end{tabular}

each respective negative control (Thermo Fisher Scientific, Waltham, MA, USA; Applied Biosystems) were transfected using Lipofectamine RNAiMAX transfection reagent (Invitrogen). Cells were plated into 6-well plates at a density of $3 \times 10^{5}$ cells/well, cultured overnight, and then transfected with $20 \mathrm{nM}$ hsa-miR-146a-5p mimic or $20 \mathrm{nM}$ hsa-miR-146a-5p inhibitor using $8 \mu \mathrm{l}$ of Lipofectamine RNAiMAX transfection reagent. After 2 days, the transfected cells were harvested and analyzed by cell viability assay, TUNEL assay and immunoblotting.

Cell viability assay. The viabilities of cells were investigated using the EZ-Cytox Cell Viability Assay kit (water-soluble tetrazolium salt method; Daeil Lab Service Co., Ltd., Seoul, Korea). Cells $\left(3 \times 10^{3}\right.$ cells $\left./ 100 \mu 1\right)$ were seeded onto a 96-well microplate, adapted overnight and then transfected with hsamiR-146a-5p mimic or anti-miR hsa-miR-146a-5p inhibitor for $48 \mathrm{~h}$ at $37^{\circ} \mathrm{C}$. The water-soluble tetrazolium salt reagent solution $(10 \mu \mathrm{l})$ was added to each well, and the plate was incubated for $2 \mathrm{~h}$ at $37^{\circ} \mathrm{C}$. The absorbance at $450 \mathrm{~nm}$ was then measured using a microplate reader.

TUNEL assay. Apoptosis was analyzed in situ by the TUNEL assay using the In Situ Cell Death Detection kit, Fluorescein (Roche Applied Science, Mannheim, German) according to the manufacturer's instructions. To ensure a representative count, each cell culture slide was divided into quarters, and at least two fields were photographed with Olympus BX50 fluorescence microscope. The number of apoptotic cells was measured by counting the TUNEL-positive cells. Average number and standard deviation were calculated from four independent experiments.

Immunoblotting. Cells $\left(1 \times 10^{6}\right)$ were plated onto $60-\mathrm{mm}$ dishes and then washed with ice-cold phosphate-buffered saline (PBS) and lysed with $150 \mu \mathrm{l}$ RIPA buffer (50 mM Tris-HCl, $\mathrm{pH} 7.4$, $150 \mathrm{mM} \mathrm{NaCl}, 1 \mathrm{mM}$ EDTA, 1\% Triton X-100, 1 mM PMSF, $2 \mathrm{mM} \mathrm{Na}_{3} \mathrm{VO}_{4}$ and $1 \mathrm{mM}$ glycerol phosphate) containing a protease inhibitor cocktail. The proteins present in the RIPA lysates were denatured using SDS sample buffer, resolved by SDS-PAGE and electroblotted onto nitrocellulose membranes. The membranes were immunoblotted with primary antibodies against Smad4; FADD; p-FADD (Ser194); NF-кB p65; JNK; p-JNK (Thr183/Tyr185); p38; phospho-p38 (Thr180/Tyr182); Bcl-2 (Cell Signaling Technology, Danvers, MA, USA); Mad (Abcam, Cambridge, MA, USA); p53 (Millipore, Bedford, MA, USA); TRAF6; TGF $\beta 1$; p15; c-Jun; p-c-Jun (Santa Cruz Biotechnology); or actin (Sigma-Aldrich). All blots were then incubated with anti-rabbit horseradish peroxidase-conjugated secondary antibodies (Cell Signaling Technology). The signals were detected by electrochemiluminescence (iNtRON Biotechnology, Seongnam-si, Korea). 

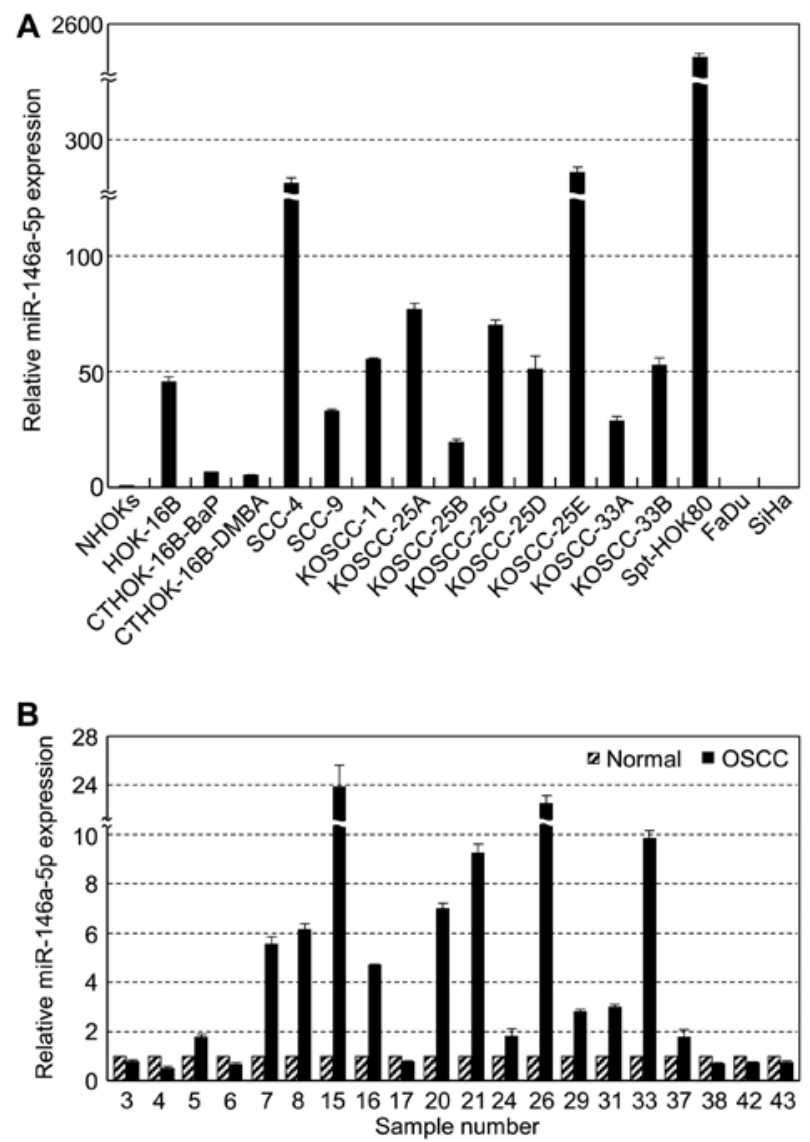
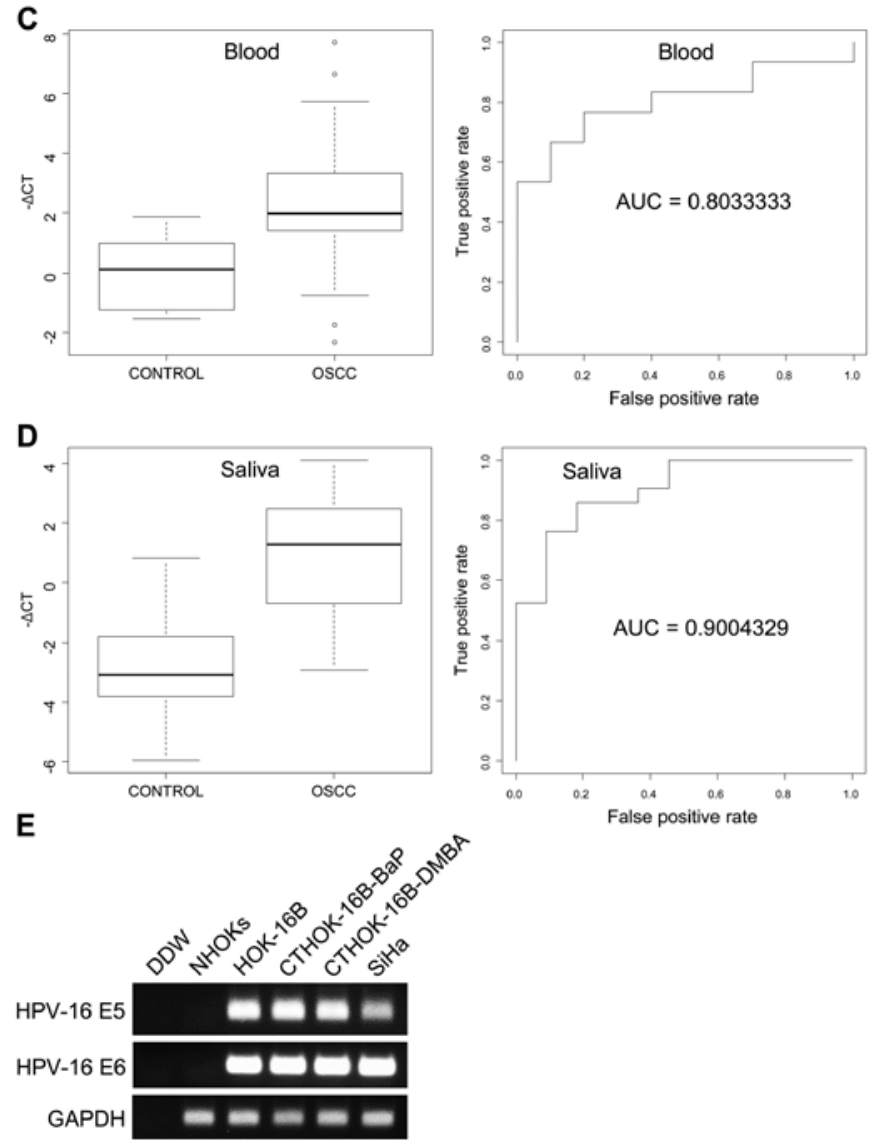

Figure 1. Expression of miR-146a-5p in OSCC cell lines and in OSCC tissues, whole blood and whole saliva of OSCC patients. (A) Relative miR-146a-5p expression levels in normal and immortalized human oral keratinocytes, 13 OSCC cell lines, and 2 SCC cell lines established from pharynx and cervix carcinomas by a TaqMan miRNA assay. (B) Relative miR-146a-5p expression levels in matched OSCC-normal tissue pairs by a TaqMan miRNA assay. The $\Delta \mathrm{CT}$ and ROC curve analyses of whole blood (C) and saliva (D) miR-146a-5p in the control individuals and in pre-operative OSCC patients. The distribution of $-\triangle \mathrm{CT}$ values in each sample was plotted as box plots for comparison and ROC curves were produced to assess their diagnostic values. (E) Determination of HPV infection and the typing of HPV by type-specific PCR analysis. NHOKs were used as a negative control for HPV infection. HOK-16B was used as positive control for HPV-16 infection.

Statistical analyses. Comparison of the distribution of $-\Delta \mathrm{CT}$ values between groups were analyzed by the language $\mathrm{R}(41)$, and ROC curves were obtained with the package ROCR (42). All other data are presented as the mean \pm SD. The statistical analyses of data were performed using the Statistica 6.0 software package (StatSoft, Tulsa, OK, USA). Results were compared using analysis of variance tests. When significant differences were found, pairwise comparisons were performed using Scheffe's adjustment. Statistical significance was also calculated using a two-tailed Student's t-test. Differences with a $\mathrm{P}<0.05$ were considered statistically significant.

\section{Results}

miR-146a-5p is highly expressed in OSCC. To explore possible miRNAs involved in the transformation of normal oral keratinocytes, we analyzed the difference in miRNA expression between NHOKs and HPV-16-immortalized human oral keratinocytes, HOK-16B, by miRNA microarray with cut-off values of 2-fold increase or decrease. Twenty-three miRNAs were upregulated while 46 miRNAs were downregulated in immortalized human oral keratinocytes compared to NHOKs (Table II). Microarray also revealed miR-3934-5p, miR-365b-5p, miR-146a-5p and miR-3138 being most abun- dant in immortalized human oral keratinocytes (Table II). On the basis of this, we hypothesized that miR-146a-5p expression is overexpressed in OSCCs and may underlie OSCC initiation and progression. Furthermore, the miR-146a-5p and its target genes were of interest, because miR-146a-5p regulates signal transduction of TGF- $\beta$, a potent antitumor cytokine, by repressing Smad4 in human cancer (43) and its biological function in carcinogenesis is not fully understood. To test our hypothesis, we first examined miR-146a-5p expression in 13 OSCC cell lines and other 2 SCC cell lines, and in matched 20 pairs of OSCC and normal tissues, 30 whole blood and 21 saliva samples from pre-operative OSCC patients and 10 healthy controls by a TaqMan miRNA assay. miR-146a-5p expression was significantly upregulated in all 13 OSCC cell lines examined compared to the normal oral keratinocytes; however, its expression in other 2 SCC cell lines established from pharynx and cervix carcinomas was extremely lower $(\sim 10 \%)$ than that observed in NHOKs (Fig. 1A). Analysis of matched OSCC-normal tissue pairs demonstrated an increase in miR-146a-5p expression in 65\% (13/20) of the OSCCs compared to the matched normal tissues (Fig. 1B). Blood miR-146a-5p levels were significantly higher in OSCC patients compared to the healthy controls with mean $-\triangle C T$ values of 2.2300363 and 0.1399096 , respectively 
A
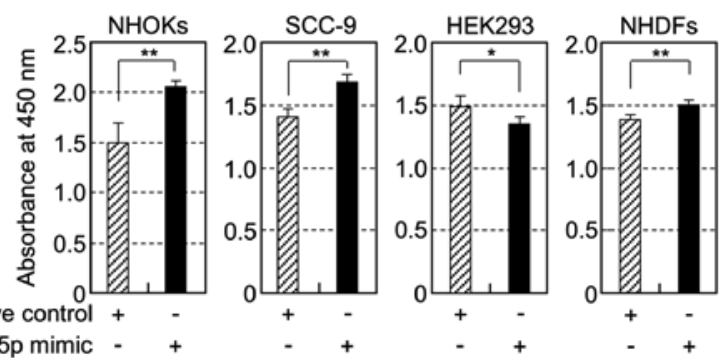

miR-146a-5p mim
B NHOKs


C

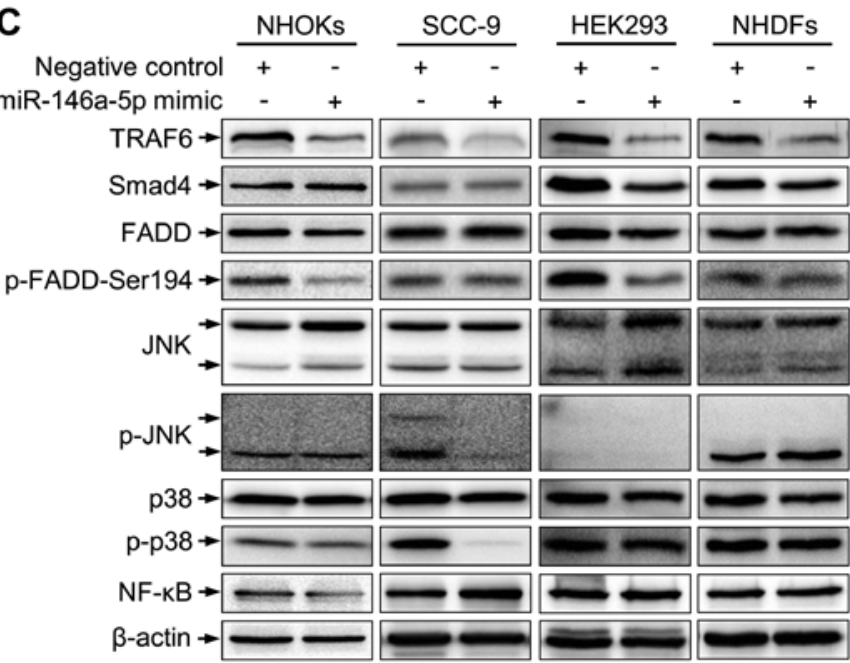

Figure 2. The effects of exogenous miR-146a-5p expression on the proliferation and apoptosis of primary NHOKs and NHDFs and SCC cell lines. (A) Cell proliferation and (B) apoptotic cells were determined in miR-146a-5p mimic-transfected cells by cell viability assay and TUNEL assay, respectively. The images in (B) are representative of two individual experiments. Scale bars, $50 \mu \mathrm{m}$. (C) Immunoblot analyses of miR-146a-5p target genes and their related genes in miR-146a-5p mimic-transfected cells. NHOKs, SCC-9, HEK293 and NHDFs were transfected with miR-146a-5p mimic (20 nM) or negative control $(20 \mathrm{nM})$ for 2 days. ${ }^{*} \mathrm{P}<0.05,{ }^{* *} \mathrm{P}<0.01$

$(\mathrm{P}=0.0007967)$ (Fig. 1C). Similarly, miR-146a-5p levels in saliva were significantly abundant in OSCC patients compared to the healthy controls with mean $-\triangle \mathrm{CT}$ values of 0.7711158 and -2.7807178, respectively ( $\mathrm{P}=0.00007308$ ) (Fig. 1D). However, among the 18 OSCC patients whose whole blood and saliva were collected, each miR-146a-5p level in blood and saliva showed minimal evidence of a true correlation when tested by the Pearson method (correlation coefficient $=-0.08920913$, $\mathrm{P}=0.7248$ ). To evaluate the diagnostic significance of miR$146 \mathrm{a}-5 \mathrm{p}$ in determining OSCC status, receiver operating characteristic (ROC) curves were obtained for each blood and saliva sample sets, and the area under the curves (AUC) was 0.8033333 and 0.9004329 , respectively (Fig. 1C and D). Collectively, these data demonstrate that miR-146a-5p is highly upregulated in immortalized human oral keratinocytes
A
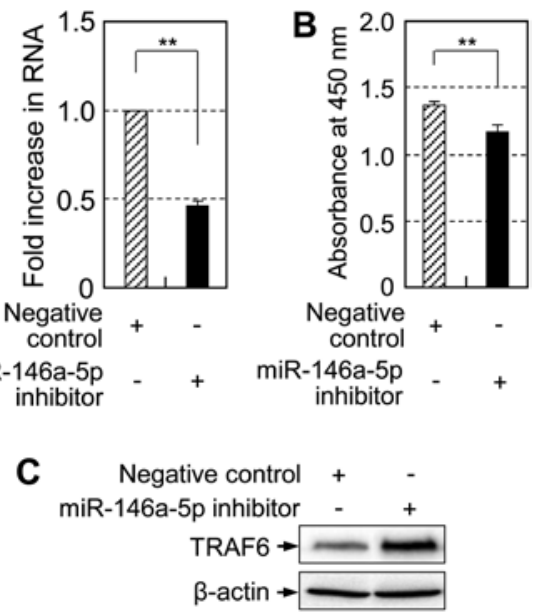

Figure 3. The effects of miR-146a-5p downregulation on cell proliferation and protein level of TRAF6 in SCC-9. (A) Relative miR-146a-5p expression level in SCC-9 after transfection with miR-146a-5p inhibitor $(40 \mathrm{nM})$ or negative control (40 $\mathrm{nM})$ by a TaqMan miRNA assay. (B) Determination of cell proliferation and immunoblot analysis of TRAF6, one of the miR-146a-5p target genes (C) in SCC-9. Cells were transfected with miR-146a-5p inhibitor $(40 \mathrm{nM})$ or negative control $(40 \mathrm{nM})$ for 2 days. ${ }^{* *} \mathrm{P}<0.01$.

and OSCC cell lines, and that miR-146a-5p levels in OSCC tissues, whole blood and whole saliva significantly increase in OSCC patients compared with healthy controls.

miR-146a-5p expression is not associated with HPV E5 in SCC cell lines. Because HPV-16 E5 oncogene is reported to modulate the expression of miR-146a-5p (44), we examined whether miR-146a-5p expression is correlated with HPV E5 expression in OSCC and SCC cell lines. We first examined whether cancer cell lines expressed 'high-risk' HPV E5 and E6 transcripts (type 16,18 and 33) and compared to their cellular miR-146a-5p levels. HPV-16 E5 and E6 transcripts were detected in HOK-16, CTHOK-16B-BaP, CTHOK-16B-DMBA and SiHa cell lines (Fig. 1E). Other 12 OSCC or SCC cell lines, however, proved to be free of HPV DNA (data not shown). The miR-146a-5p expression was significantly upregulated in HOK-16B, CTHOK-16B-BaP and CTHOK-16B-DMBA, which expressed HPV-16 E5; however, its expression in $\mathrm{SiHa}$, which expressed HPV-16 E5, was extremely lower ( 10\%) than that observed in NHOKs (Fig. 1A). Unexpectedly, our data support that miR-146a-5p expression is not necessarily correlated with HPV-16 E5 expression in human cancer cell lines.

miR-146a-5p variously affects cell proliferation and apoptosis in normal and SCC cells. In view of the observed widespread upregulation of miR-146a-5p expression in OSCC cell lines and OSCC clinical specimens, we assessed whether miR-146a-5p upregulation has associated proliferation potential in NHOKs, SCC-9, HEK293 and NHDFs. Compared to that in negative control-transfected cells, the proliferation of NHOKs and SCC-9 was significantly enhanced by introduction of exogenous miR-146a-5p (Fig. 2A), which was verified by a TaqMan miRNA assay (data not shown). Additionally, NHDFs responded with small but significant increase in cell proliferation. Distinctively, exogenous miR-146a-5p expression inhibited the proliferation of HEK293 cell line (Fig. 2A). 

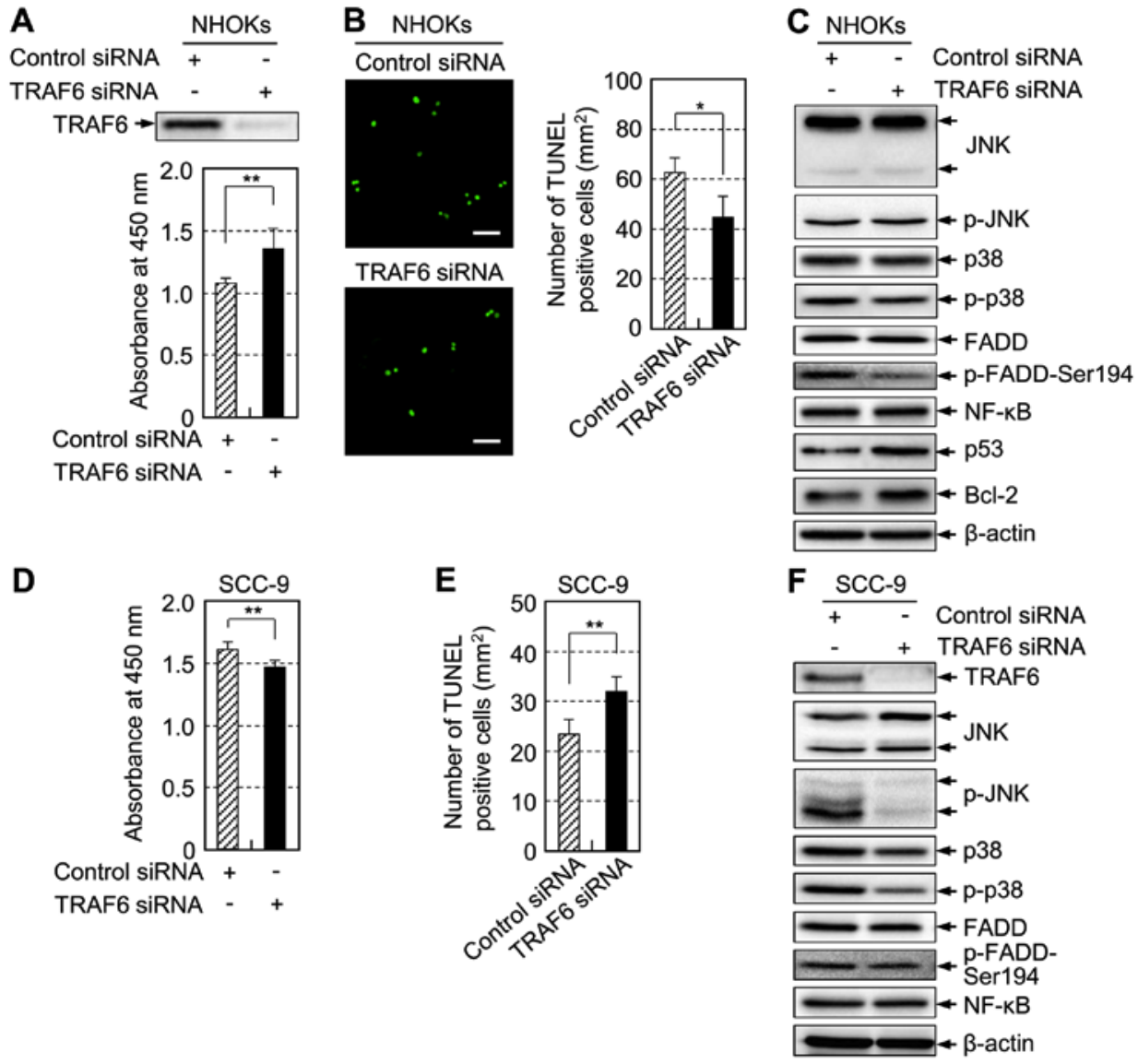

Figure 4. Suppression of TRAF6 is responsible for the effects of miR-146a-5p. (A and D) Immunoblot analysis of TRAF6 (A, upper panel) and proliferation (A, lower panel and D) in NHOKs (A) and SCC-9 (D) that were transfected with control siRNA or TRAF6-specific siRNA (40 nM). (B and E) Apoptotic cells in control siRNA- or TRAF6-specific siRNA-transfected NHOKs (B) and SCC-9 (E). (C and F) Immunoblot analyses of downstream signaling molecules of TRAF6 in control siRNA- or TRAF6-specific siRNA-transfected NHOKs (C) and SCC-9 (F). ${ }^{*} \mathrm{P}<0.05,{ }^{* *} \mathrm{P}<0.01$.

Furthermore, we determined whether high miR-146a-5p expression is associated with apoptosis in NHOKs and SCC-9 using TUNEL assay. Exogenous miR-146a-5p expression caused inhibition of apoptosis in NHOKs, while it increased apoptosis in SCC-9 (Fig. 2B). Collectively, overexpression of miR-146a-5p by transfection of cells with miR-146a-5p mimic resulted in diverse effects on proliferation and apoptosis depending on the cell types.

miR-146a-5p targets TRAF6 but not Smad4 or FADD in normal and SCC cells. To elucidate the underlying mechanisms associated with the different outcomes of miR-146a-5p on proliferation and apoptosis in different cell types, we examined the protein levels of several known miR-146a-5p target genes, such as TRAF6, SMAD4 and FADD and their downstream signaling molecules by immunoblotting. After transfection of cells with miR-146a-5p mimic, TRAF6 expression was invariably downregulated in all cell types tested; however, Smad4 expression remained constant in NHOKs and SCC-9, while it decreased in HEK293 and NHDFs (Fig. 2C). FADD expression was slightly downregulated only in HEK293 cells (Fig. 2C). Notably, exogenous miR-146a-5p expression downregulated phosphorylated FADD at Ser194 (p-FADDSer194) in NHOKs and HEK293 cells but not in SCC-9 and NHDFs (Fig. 2C). JNK and p38 are MAPKs at the downstream of TRAF6 in TGF- $\beta$ signaling that are both activated by phosphorylation (27). Exogenous miR-146a-5p expression deactivated JNK in SCC-9, but had no discernable effects in NHOKs, HEK293 and NHDFs (Fig. 2C). Similarly, exogenous miR-146a-5p expression also deactivated p38 in SCC-9. These data suggest that miR-146a-5p hampers translation of its target genes in a cell type-specific fashion.

miR-146a-5p confers proliferation advantage to SCC-9. In the opposite approach, we suppressed endogenous miR-146a-5p expression in SCC-9 followed by functional assays. Suppression $(\sim 46 \%)$ of endogenous miR-146a-5p expression (Fig. 3A) by transfection of cells with miR-146a-5p inhibitor significantly inhibited cell proliferation compared to the negative control-transfected cells (Fig. 3B). SCC-9 rather than SCC-4 was selected for the further studies to clearly elucidate the effects of miR-146a-5p on cellular processes of OSCC cell lines in this study. It is important to finely control the expression levels of miR-146a-5p in the cell lines by its mimic or inhibitor. Since the basal miR-146a-5p expression level in SCC-4 was too high (Fig. 1A), 54\% reduction of its expression by the miR-146a-5p inhibitor, as shown in Fig. 3A, seemed still high, making it ambiguous to clarify its reduced function. On the other hand, SCC-9 had relatively lower, but significantly higher expression level of miR-146a-5p compared to normal oral keratinocytes (Fig. 1A), making it more effective to examine the clear effect of the miR-146a-5p 



Figure 5. The effects of SP600125 and SB203580 on cellular processes and expression of TRAF6 and its downstream signaling molecules. (A) Determination of proliferation and (B) apoptotic cells in exponentially proliferating NHOKs treated with SP600125, a JNK specific inhibitor, for 1 day. (C) Determination of proliferation and (D) immunoblot analyses of TRAF6 and its downstream signaling molecules in SCC-9 treated with SP600125 for 1 day. (E) Determination of proliferation in exponentially proliferating NHOKs and (F) SCC-9 treated with SB203580, a p38 specific inhibitor, for 1 day. (G) Immunoblot analyses of TRAF6 and its downstream signaling molecules in SCC-9 treated with SB203580 for 1 day. (H) Effects of TGF- $\beta 1$ on the protein levels of TRAF6-mediated branch of the TGF- $\beta$ signaling in NHOKs. Exponentially proliferating NHOKs were treated with $20 \mathrm{ng} / \mathrm{ml}$ TGF- $\beta 1$ for 2 days. ${ }^{* *} \mathrm{P}<0.01$.

mimic or inhibitor transfection. Next, to assess the underlying mechanisms of miR-146a-5p inhibitor-induced inhibition of cell proliferation, we examined the protein level of TRAF6, one of the miR-146a-5p target genes. miR-146a-5p inhibitor induced upregulation of TRAF6 (Fig. 3C). Our data suggest that miR-146a-5p inhibitor inhibits proliferation of SCC-9 by modulating the expression level of TRAF6.

TRAF6 silencing induces deactivation of JNK and p38 in SCC-9 but not in NHOKs. To assess the functional significance of the universal effect of exogenous miR-146a-5p expression, which led to TRAF6 downregulation, we silenced endogenous TRAF6 expression in exponentially proliferating NHOKs and SCC-9 by transfecting the cells with a specific small interfering RNA (siRNA). TRAF6 knockdown (Fig. 4A, upper panel) led to significantly increased cell proliferation (Fig. 4A, lower panel) and inhibited apoptosis of NHOKs (Fig. 4B), mimicking the effects of exogenous miR-146a-5p expression (Fig. 2A and B). By contrast, TRAF6 knockdown significantly decreased cell proliferation (Fig. 4D) and increased apoptosis (Fig. 4E) of SCC-9. Immunoblotting revealed that TRAF6 knockdown resulted in marked deactivation of JNK and p38 in SCC-9 (Fig. 4F), giving very similar results to those of the exogenous miR-146a-5p expression (Fig. 2C). However, TRAF6 knockdown did not affect the phosphorylation of JNK and p38 but decreased the amount of p-FADD-Ser194 in NHOKs (Fig. 4C). Overall, our data suggest two points; first, TRAF6 knockdown can be either proliferative or inhibitory in terms of cell proliferation depending on the cellular context, and second, the TRAF6-JNK and TRAF6-p38 channels can be constitutively active in SCC-9.

JNK plays a crucial role in the proliferation of NHOKs and $S C C-9$. The phenotypically contradictory results of TRAF6 silencing imply antagonistic effectors downstream of TRAF6 signaling. Since JNK and p38 are activated by TRAF6 in certain circumstances, we defined their roles using specific kinase inhibitors targeting each of them. Treatment of NHOKs with SP600125, a JNK specific inhibitor, significantly reduced cell proliferation (Fig. 5A) and increased apoptosis (Fig. 5B) in a dose-dependent manner, as well as reduced proliferation of SCC-9, similarly (Fig. 5C). Immunoblotting revealed that SP600125 inhibited phosphorylation of JNK and its substrate c-Jun in a dose-dependent manner; compellingly, p-FADD-Ser194 was also reduced in SCC-9 (Fig. 5D). By contrast, SB203580, a p38 inhibitor, resulted in an insignificant 

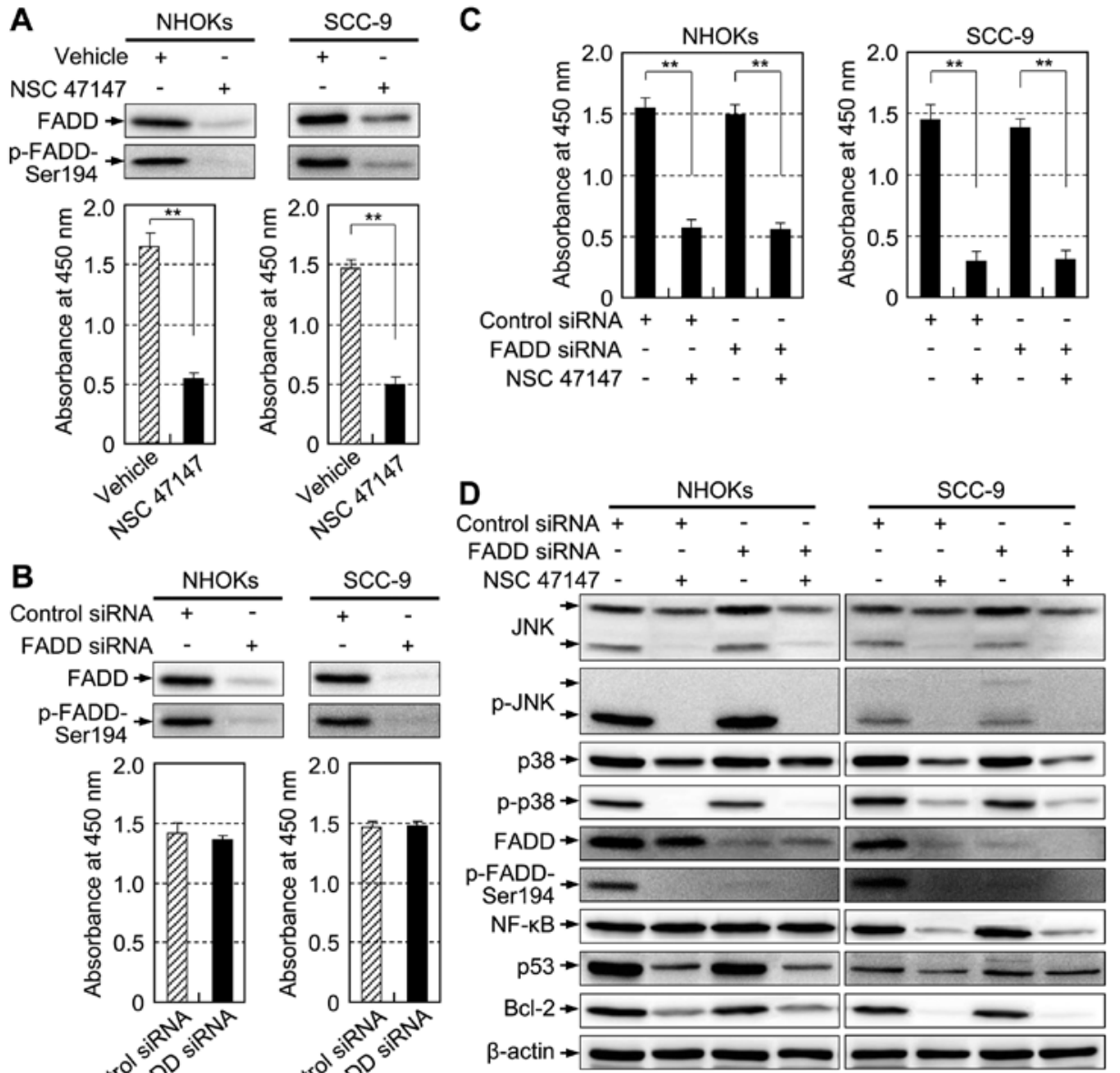

Figure 6. The effects of NSC 47147 and FADD silencing on cell proliferation. (A) Immunoblot analyses of FADD and p-FADD-Ser194 (upper panel) and proliferation (lower panel) in NHOKs and SCC-9 that were treated with NSC $47147(6 \mu \mathrm{M})$, a kinase inhibitor which inhibits FADD phosphorylation, for 1 day. (B) Immunoblot analyses of FADD and p-FADD-Ser194 (upper panel) and proliferation (lower panel) in NHOKs and SCC-9 that were transfected with control siRNA or FADD-specific siRNA (40 nM). (C and D) Effects of NSC 47147 on proliferation (C) and expression of signaling molecules (D) in FADD-specific siRNA-transfected cells. Exponentially proliferating NHOKs and SCC-9 transfected with control siRNA (40 nM) or FADD-specific siRNA (40 nM) for 2 days were further treated with $6 \mu \mathrm{M}$ NSC 47147 for 1 day. ${ }^{* *} \mathrm{P}<0.01$.

reduction of proliferation of NHOKs (Fig. 5E) but reduced proliferation of SCC-9 (Fig. 5F). Unexpectedly, reduction of phosphorylated p38 was marginal, and an unanticipated upregulation of JNK and phosphorylated JNK was observed in SCC-9 by immunoblotting. JNK activation by SB203580 also increased phosphorylated c-Jun (Fig. 5G). Similarly, TGF- $\beta 1$ activated JNK and c-Jun, but not p38 in exponentially proliferating NHOKs (Fig. 5H). These likely secondary effects could have dampened the effects of $\mathrm{p} 38$ suppression on cell proliferation. Nevertheless, these data suggest that JNK/c-Jun signaling pathway plays a key role in regulation of proliferation of SCC-9.

FADD and $p-F A D D$ are not involved in the proliferation of NHOKs and SCC-9. Since exogenous miR-146a-5p expression reduced the expression of p-FADD-Ser194 in NHOKs and HEK293 (Fig. 2C), we opted to investigate the functional relevance of p-FADD-Ser194 in cell proliferation. Proliferation was drastically inhibited by treatment of NHOKs and SCC-9 with NSC 47147 (Fig. 6A, lower panel), a kinase inhibitor that is known to inhibit FADD phosphorylation (45). Notably, expression of both FADD and p-FADD was suppressed (Fig. 6A, upper panel). Thus, to confirm the effects of simultaneous downregulation of both FADD and p-FADD-Ser194 expression, we suppressed their expression by transfection of cells with a FADD-specific siRNA. Unexpectedly, simultaneous silencing of both FADD and p-FADD-Ser194 (Fig. 6B, upper panel) did not result in cell proliferation blockage of NHOKs and SCC-9 (Fig. 6B, lower panel). Therefore, to verify these conflicting results, cells transfected with a FADD-specific siRNA were subsequently treated with NSC 47147. NSC 47147 was able to reduce cell proliferation in the absence of FADD and p-FADD-Ser194 expression, and the inhibition pattern of cell proliferation was strikingly similar to that of control siRNA-transfected cells (Fig. 6C). Immunoblotting revealed that NSC 47147 not only inhibited phosphorylation of FADD, but also inhibited phosphorylation of JNK and p38, suggesting a broad inhibition of MAPK pathway (Fig. 6D). Overall, our data suggest that FADD and p-FADD-Ser194 expression has a minimal effect on the proliferation of NHOKs and SCC-9.

\section{Discussion}

Overexpression of miR-146a-5p has been previously identified in OSCC tissues $(7,46)$, blood of OSCC patients (7), and oral precancerous lesions (46). In the present study, we widened 
the view with salivary abundance of the miR-146a-5p. The miR-146a-5p in saliva seemed not to originate from blood, which was suggested by the poor correlation of $-\Delta C T$ values in matched samples. The tumor could contribute to miR-146a-5p levels in blood and saliva, respectively, by direct shedding of the miRNA. Both levels of miR-146a-5p in blood and saliva of OSCC patients proved themselves to be a possible candidate as a diagnostic marker of OSCC $(47,48)$. However, clinical application of miR-146a-5p as a diagnostic marker requires further validation, because disease status other than OSCC, such as oral lichen planus and psoriasis, may also have high levels of miR-146a-5p in their blood or saliva (49).

miR-146a-5p was highly upregulated in immortalized human oral keratinocytes and all the OSCC cell lines examined, but not in other SCC cell lines. Although upregulation of miR-146 in cervial carcinomas is previously reported (5), its expression was almost negligible in SiHa cell line established from cervical carcinoma. This is supported by the fact that all seven cervical cancer cell lines examined expressed no detectable miR-146a (5). In addition, differently from a previous study (44), we found that miR-146a-5p expression is not necessarily correlated with 'high-risk' HPV E5 expression in human cancer cell lines. These findings partially explain the discrepancy between low incidence of HPV and overexpression of miR-146a-5p in OSCC. Otherwise, contributing factors of miR-146a-5p upregulation possibly includes cytokines that activate NF- $\kappa$ B. TGF- $\beta$ upregulated miR-146a-5p expression in NHOKs (50), which may also contribute to the overexpression of the miRNA in OSCC. Other possible candidates include tumor necrosis factor and IL-1. Thus, overexpression of miR-146a-5p in OSCC cell lines may reflect the constitutive activation of $\mathrm{NF}-\kappa \mathrm{B}$ in these cells.

Despite of its wide involvement in cell biology and increasing research, miRNAs are getting more and more thrown into disarray owing to their functionally complicated nature. miR-146a/b has been previously reported to be linked with cancer risk and invasive and metastatic capacity in diverse cancers (3); however, its roles in carcinogenesis are not fully understood yet. In the present study, we demonstrated two additional layers of complexity that require attention to apprehend the significance of the miRNA. The first layer of miRNA complexity comprised of cell type- and/or contextdependent miRNA target selection, which resulted in sparing of a certain target while suppressing another universally. For instance, the range of target mRNAs of a single miRNA such as miR-146a-5p differed by cell type. The gold standard for miRNA functionality is verification of the protein expression pattern of target mRNAs. Indeed, our data demonstrated that a validated miRNA target in a specific cell type can dodge from the miRNA-mediated post-transcriptional suppression in another cell type. This evasion from post-transcriptional suppression by miRNA could impart variable contexts that phenomenalize the effects of a miRNA to the cells expressing it.

Specifically, for human oral keratinocytes, miR-146a-5p spared Smad4 from post-transcriptional suppression. Smad4 plays a crucial role in the canonical TGF- $\beta$ signaling pathway and is implicated in epithelial-mesenchymal transition by TGF- $\beta$ (51). A considerable body of evidence indicates that Smad4 is a valid target gene of miR-146a-5p $(22,43)$; however, exogenous miR-146a-5p expression did not affect the expression levels of Smad4 in NHOKs and SCC cell lines. This observation suggests that miR-146a-5p selectively disarms the non-canonical, TRAF6-mediated branch of the TGF- $\beta$ signaling in human oral keratinocytes. One theoretical consequence of sparing Smad4 involvement in human oral keratinocytes is that the discriminative downregulation by miR-146a-5p may selectively confer resistance against cytostatic and pro-apoptotic effect of TGF- $\beta$ signaling, while preserving other arms of the pathway, possibly the epithelialmesenchymal transition inducing arm, to the cells. Future studies need to address this possibility to clarify the significance of miR-146a-5p upregulation on metastasis.

The second layer of miRNA complexity arose from the functional pleiotropism of a miRNA target gene, which in this study was exemplified by TRAF6. TRAF6 was a universal target of miR-146a-5p in all cells and cell lines tested; however, TRAF6 suppression by a TRAF6-specific siRNA resulted in contradictory functional consequences in NHOKs and SCC-9. There seemed to be conflicting downstream signals under the common signal transducer TRAF6, which comprised of a proliferative signal exemplified by JNK in SCC-9 and an anti-proliferative signal that suppress the expression of $\mathrm{Bcl}-2$ in NHOKs. Our results from TRAF6-specific siRNA experiments showed that the JNK arm is dependent on TRAF6 in SCC-9 but not in NHOKs. Namely, TRAF6 downregulation by both miR-146a-5p and TRAF6-specific siRNA deactivated JNK in SCC-9 but not in NHOKs. Nevertheless, the causative signaling that constitutively activates TRAF6-mediated JNK activity is currently undefined. One of the possible candidates seems to be TGF- $\beta$ signaling, because TRAF6 suppression is implicated in the abrogation of TGF- $\beta$ signaling by miR-146a-5p (50). TGF- $\beta$ signaling encompasses the MAPK pathway, which starts from the interaction between TRAF6 and TAK1 and results in JNK activation $(26,27)$. As expected, we observed that TGF- $\beta 1$ activated JNK and its substrate c-Jun in exponentially proliferating NHOKs.

FADD expression was not affected by miR-146a-5p in all cells and cell lines tested. This represented additional support to the functional diversity of the miR-146a-5p, because FADD expression was repressed by the miRNA in HEK293. Since miR-146a-5p reduced the proportion of $p-F A D D$ in several cells, we tested the function of FADD and p-FADD in proliferation of NHOKs and SCC-9. We showed that suppression of both FADD and p-FADD by combined treatment of FADD-specific siRNA and NSC 47147 was not implicated in proliferation control, at least in steady state of NHOKs and SCC-9. Rather, the anti-proliferative effect of NSC 47147 was associated with inactivation of JNK, which further support the crucial role of the kinase in proliferation of NHOKs and SCC-9. Further studies are required to verify the role of periodontitis in miR-146a-5p expression in oral cancer patients, and to investigate the role of miR-146a-5p in oral cancer using in vivo animal models.

In summary, our findings showed that miR-146a-5p was upregulated in OSCC and granted proliferative advantage to normal oral keratinocytes and OSCC cell lines in a contextdependent manner. Our data also suggested that miR-146a-5p selectively disarmed the TRAF6-mediated branch of the 
TGF- $\beta$ signaling in OSCC cell lines by sparing Smad4 involvement.

\section{Acknowledgements}

The present study was supported by the Midcareer Researcher Program through agrant from the National Research Foundation of Korea, funded by the Ministry of Science, ICT and Future Planning, Korean government (2016R1A2B2007246), and the Korea Healthcare Technology R\&D Project, funded by the Ministry for Health, Welfare \& Family Affairs, Republic of Korea (HI15C2455) (to B.-M.M.).

\section{References}

1. Calin GA and Croce CM: MicroRNA signatures in human cancers. Nat Rev Cancer 6: 857-866, 2006.

2. Svoronos AA, Engelman DM and Slack FJ: OncomiR or tumor suppressor? The duplicity of microRNAs in cancer. Cancer Res 76: 3666-3670, 2016.

3. Elsarraj HS, Stecklein SR, Valdez K and Behbod F: Emerging functions of microRNA-146a/b in development and breast cancer: microRNA-146a/b in development and breast cancer. J Mammary Gland Biol Neoplasia 17: 79-87, 2012.

4. Chang SS, Jiang WW, Smith I, Poeta LM, Begum S, Glazer C, Shan S, Westra W, Sidransky D and Califano JA: MicroRNA alterations in head and neck squamous cell carcinoma. Int $\mathrm{J}$ Cancer 123: 2791-2797, 2008.

5. Wang X, Tang S, Le SY, Lu R, Rader JS, Meyers C and Zheng ZM: Aberrant expression of oncogenic and tumor-suppressive microRNAs in cervical cancer is required for cancer cell growth. PLoS One 3: e2557, 2008.

6. Raponi M, Dossey L, Jatkoe T, Wu X, Chen G, Fan H and Beer DG: MicroRNA classifiers for predicting prognosis of squamous cell lung cancer. Cancer Res 69: 5776-5783, 2009.

7. Hung PS, Liu CJ, Chou CS, Kao SY, Yang CC, Chang KW, Chiu TH and Lin SC: miR-146a enhances the oncogenicity of oral carcinoma by concomitant targeting of the IRAK1, TRAF6 and NUMB genes. PLoS One 8: e79926, 2013.

8. Hurst DR, Edmonds MD, Scott GK, Benz CC, Vaidya KS and Welch DR: Breast cancer metastasis suppressor 1 up-regulates miR-146, which suppresses breast cancer metastasis. Cancer Res 69: 1279-1283, 2009.

9. Kogo R, Mimori K, Tanaka F, Komune S and Mori M: Clinical significance of miR-146a in gastric cancer cases. Clin Cancer Res 17: 4277-4284, 2011.

10. Lin SL, Chiang A, Chang D and Ying SY: Loss of mir-146a function in hormone-refractory prostate cancer. RNA 14: 417-424, 2008.

11. Li Y, Vandenboom TG II, Wang Z, Kong D, Ali S, Philip PA and Sarkar FH: miR-146a suppresses invasion of pancreatic cancer cells. Cancer Res 70: 1486-1495, 2010.

12. Kloosterman WP and Plasterk RHA: The diverse functions of microRNAs in animal development and disease. Dev Cell 11: 441-450, 2006.

13. Marques-Rocha JL, Samblas M, Milagro FI, Bressan J, Martínez JA and Marti A: Noncoding RNAs, cytokines, and inflammation-related diseases. FASEB J 29: 3595-3611, 2015.

14. O'Connell RM, Rao DS and Baltimore D: microRNA regulation of inflammatory responses. Annu Rev Immunol 30: 295-312, 2012.

15. Kundu JK and Surh YJ: Inflammation: Gearing the journey to cancer. Mutat Res 659: 15-30, 2008

16. Perry MM, Moschos SA, Williams AE, Shepherd NJ, LarnerSvensson HM and Lindsay MA: Rapid changes in microRNA-146a expression negatively regulate the IL- $1 \beta$-induced inflammatory response in human lung alveolar epithelial cells. J Immunol 180 5689-5698, 2008.

17. Liu X, Nelson A, Wang X, Kanaji N, Kim M, Sato T, Nakanishi M, Li Y, Sun J, Michalski J, et al: MicroRNA-146a modulates human bronchial epithelial cell survival in response to the cytokineinduced apoptosis. Biochem Biophys Res Commun 380: 177-182, 2009.

18. Xie YF, Shu R, Jiang SY, Liu DL and Zhang XL: Comparison of microRNA profiles of human periodontal diseased and healthy gingival tissues. Int J Oral Sci 3: 125-134, 2011.
19. Irwandi RA and Vacharaksa A: The role of microRNA in periodontal tissue: A review of the literature. Arch Oral Biol 72: 66-74, 2016.

20. Olsen I, Singhrao SK and Osmundsen H: Periodontitis, pathogenesis and progression: miRNA-mediated cellular responses to Porphyromonas gingivalis. J Oral Microbiol 9: 1333396, 2017.

21. Preedy VR and Hunter RJ: Cytokine. In: MicroRNAs and Cytokines. Liu XD (ed). CRC Press, New York, pp10-16, 2011.

22. Xiao B, Zhu ED, Li N, Lu DS, Li W, Li BS, Zhao YL, Mao XH, Guo G, Yu PW, et al: Increased miR-146a in gastric cancer directly targets SMAD4 and is involved in modulating cell proliferation and apoptosis. Oncol Rep 27: 559-566, 2012.

23. Paik JH, Jang JY, Jeon YK, Kim WY, Kim TM, Heo DS and Kim CW: MicroRNA-146a downregulates NF- $\kappa$ B activity via targeting TRAF6 and functions as a tumor suppressor having strong prognostic implications in NK/T cell lymphoma. Clin Cancer Res 17: 4761-4771, 2011.

24. Massagué J, Blain SW and Lo RS: TGFbeta signaling in growth control, cancer, and heritable disorders. Cell 103: 295-309, 2000.

25. Zhang YE: Non-Smad pathways in TGF- $\beta$ signaling. Cell Res 19: 128-139, 2009.

26. Sorrentino A, Thakur N, Grimsby S, Marcusson A, von Bulow V, Schuster N, Zhang S, Heldin CH and Landström M: The type I TGF- $\beta$ receptor engages TRAF6 to activate TAK1 in a receptor kinase-independent manner. Nat Cell Biol 10: 1199-1207, 2008.

27. Yamashita M, Fatyol K, Jin C, Wang X, Liu Z and Zhang YE: TRAF6 mediates Smad-independent activation of JNK and p38 by TGF- $\beta$. Mol Cell 31: 918-924, 2008.

28. Wagner EF and Nebreda AR: Signal integration by JNK and p38 MAPK pathways in cancer development. Nat Rev Cancer 9: 537-549, 2009.

29. Vaiopoulos AG, Papachroni KK and Papavassiliou AG: Colon carcinogenesis: Learning from NF- $\mathrm{KB}$ and AP-1. Int J Biochem Cell Biol 42: 1061-1065, 2010.

30. He L, Wu X, Siegel R and Lipsky PE: TRAF6 regulates cell fate decisions by inducing caspase 8-dependent apoptosis and the activation of NF- $\mathrm{BB}$. J Biol Chem 281: 11235-11249, 2006.

31. Peter ME and Krammer PH: Mechanisms of CD95 (APO-1/Fas)mediated apoptosis. Curr Opin Immunol 10: 545-551, 1998.

32. Curtale G, Citarella F, Carissimi C, Goldoni M, Carucci N, Fulci V, Franceschini D, Meloni F, Barnaba V and Macino G: An emerging player in the adaptive immune response: microRNA146a is a modulator of IL-2 expression and activation-induced cell death in T lymphocytes. Blood 115: 265-273, 2010.

33. Chen G, Bhojani MS, Heaford AC, Chang DC, Laxman B, Thomas DG, Griffin LB, Yu J, Coppola JM, Giordano TJ, et al: Phosphorylated FADD induces NF- $\kappa \mathrm{B}$, perturbs cell cycle, and is associated with poor outcome in lung adenocarcinomas. Proc Natl Acad Sci USA 102: 12507-12512, 2005.

34. Hua ZC, Sohn SJ, Kang C, Cado D and Winoto A: A function of Fas-associated death domain protein in cell cycle progression localized to a single amino acid at its $\mathrm{C}$-terminal region. Immunity 18: 513-521, 2003.

35. Oh JE, Kook JK and Min BM: ßig-h3 induces keratinocyte differentiation via modulation of involucrin and transglutaminase expression through the integrin $\alpha 3 \beta 1$ and the phosphatidylinositol 3-kinase/Akt signaling pathway. J Biol Chem 280: 21629-21637, 2005.

36. Yeo IS, Oh JE, Jeong L, Lee TS, Lee SJ, Park WH and Min BM: Collagen-based biomimetic nanofibrous scaffolds: Preparation and characterization of collagen/silk fibroin bicomponent nanofibrous structures. Biomacromolecules 9: 1106-1116, 2008.

37. Park NH, Min BM, Li SL, Huang MZ, Cherick HM and Doniger J: Immortalization of normal human oral keratinocytes with type 16 human papillomavirus. Carcinogenesis 12: 1627-1631, 1991.

38. Min B, Woo K, Baek J, Lee G and Park N: Malignant transformation of hpv-immortalized human oral keratinocytes by chemical carcinogens. Int J Oncol 7: 249-256, 1995.

39. Jang DH, Oh JE, Kang HK, Kim OB, Min SK, Jung SY, Hong SD, Lee JI and Min BM: Spontaneous tumorigenicity of primary human oral keratinocytes with human papillomavirus negativity and impaired apoptosis. Int J Oncol 36: 1491-1501, 2010.

40. Lee G, Kim YB, Kim JH, Kim MS, Shin KH, Won YS, Lee JI, Choung PH, Hyun BH and Min BM: Characterization of novel cell lines established from three human oral squamous cell carcinomas. Int J Oncol 20: 1151-1159, 2002. 
41. R Development Core Team (2011), R: A Language and Environment for Statistical Computing. Vienna, Austria : the R Foundation for Statistical Computing. ISBN: 3-900051-07-0. Available online at http://www.R-project.org/.

42. Sing T, Sander O, Beerenwinkel N and Lengauer T: ROCR: Visualizing classifier performance in R. Bioinformatics 21: 3940-3941, 2005.

43. Geraldo MV, Yamashita AS and Kimura ET: MicroRNA miR-146b-5p regulates signal transduction of TGF- $\beta$ by repressing SMAD4 in thyroid cancer. Oncogene 31: 1910-1922, 2012.

44. Greco D, Kivi N, Qian K, Leivonen SK, Auvinen P and Auvinen E: Human papillomavirus 16 E5 modulates the expression of host microRNAs. PLoS One 6: e21646, 2011.

45. Schinske KA, Nyati S, Khan AP, Williams TM, Johnson TD, Ross BD, Tomás RP and Rehemtulla A: A novel kinase inhibitor of FADD phosphorylation chemosensitizes through the inhibition of NF- $\kappa$ B. Mol Cancer Ther 10: 1807-1817, 2011.

46. Cervigne NK, Reis PP, Machado J, Sadikovic B, Bradley G, Galloni NN, Pintilie M, Jurisica I, Perez-Ordonez B, Gilbert R, et al: Identification of a microRNA signature associated with progression of leukoplakia to oral carcinoma. Hum Mol Genet 18: 4818-4829, 2009.
47. Mitchell PS, Parkin RK, Kroh EM, Fritz BR, Wyman SK, Pogosova-Agadjanyan EL, Peterson A, Noteboom J, O'Briant KC, Allen A, et al: Circulating microRNAs as stable blood-based markers for cancer detection. Proc Natl Acad Sci USA 105: 10513-10518, 2008.

48. Park NJ, Zhou H, Elashoff D, Henson BS, Kastratovic DA, Abemayor E and Wong DT: Salivary microRNA: Discovery, characterization, and clinical utility for oral cancer detection. Clin Cancer Res 15: 5473-5477, 2009.

49. Arão TC, Guimarães ALS, de Paula AMB, Gomes CC and Gomez RS: Increased miRNA-146a and miRNA-155 expressions in oral lichen planus. Arch Dermatol Res 304: 371-375, 2012.

50. Min SK, Jung SY, Kang HK, Jo SB, Kim MJ and Min BM: MicroRNA-146a-5p limits elevated TGF- $\beta$ signal during cell senescence. Mol Ther Nucleic Acids 7: 335-338, 2017.

51. Massagué J: TGFbeta in cancer. Cell 134: 215-230, 2008. 